\title{
Prediction model based on blood urea nitrogen and the leukocyte count for intestinal necrosis in patients with portal vein system thrombosis: a retrospective study
}

\author{
Qiang Wei, Zenglei He, Kun Wang, Changbiao Li, Abdulahad Al Ameri, Shusen Zheng, Xiao Xu \\ Department of Hepatobiliary and Pancreatic Surgery, First Affiliated Hospital, Zhejiang University School of Medicine, Hangzhou 310003 , China \\ Contributions: (I) Conception and design: Q Wei, X Xu; (II) Administrative support: X Xu, S Zheng, Z He, K Wang; (III) Provision of study materials \\ or patients: Q Wei, Z He; (IV) Collection and assembly of data: C Li, A Al Ameri; (V) Data analysis and interpretation: K Wang; (VI) Manuscript \\ writing: All authors; (VII) Final approval of manuscript: All authors. \\ Correspondence to: Prof. Xiao Xu, MD, PhD. Department of Hepatobiliary and Pancreatic Surgery, First Affiliated Hospital, Zhejiang University \\ School of Medicine, 79 Qingchun Road, Hangzhou 310003, China. Email: zjxu@zju.edu.cn.
}

\begin{abstract}
Background: Portal vein system thrombosis (PVST) is a serious and potentially fatal disease. No definite parameter can predict intestinal necrosis in patients with PVST to justify early surgical intervention. The current study aimed to explore a simple and accurate model to predict the occurrence of intestinal necrosis in patients with PVST.

Methods: Records of patients admitted to our emergency department with PVST from January 2010 to October 2018 were reviewed. Clinical parameters, including patient history, physical examination, and the results of laboratory investigations, were analyzed.

Results: Sixty-nine patients (27 females) were included. All patients were admitted to our emergency department because of abdominal pain. Fourteen patients required exploratory laparotomy, and intestinal necrosis was confirmed. Seven patients received thrombolytic therapy, and the other 48 patients had completed anticoagulation successfully. According to multivariate logistic regression, high blood urea nitrogen (BUN) (OR: 1.413, P=0.048) and the leukocyte count (OR: 1.180, $\mathrm{P}=0.005)$ were associated with intestinal necrosis, and a prediction model for intestinal necrosis (PMIN) based on the BUN and leukocyte count was established.
\end{abstract}

Conclusions: The PMIN score could effectively predict intestinal necrosis in patients with PVST.

Keywords: Model; intestine; necrosis; portal vein; thrombosis

Submitted Dec 07, 2019. Accepted for publication Feb 05, 2020.

doi: $10.21037 /$ atm.2020.02.108

View this article at: http://dx.doi.org/10.21037/atm.2020.02.108

\section{Introduction}

Portal vein system thrombosis (PVST), including portal vein thrombosis (PVT) and superior mesenteric vein thrombosis (SMVT), is a potentially life-threatening condition that has a concealed onset without any specific symptoms and signs $(1,2)$. The prevalence of PVST ranges from $10-23 \%$ or approximately $1 \%$ in patients with or without liver cirrhosis, respectively $(3,4)$. PVST often leads to an increase in mesenteric venous pressure and subsequent congestion of the intestinal wall, which possibly results in transmural necrosis of the bowel wall. Thus, early diagnosis and timely treatment are very important. In addition, the location of thrombosis is often associated with the severity of clinical symptoms. For instance, if thrombosis occurs only in the portal vein, clinical symptoms such as abdominal pain are usually not serious, whereas thrombosis of the superior mesenteric vein presents with the upper middle or lower middle abdomen, nausea, vomiting, and/or hematochezia (5). Many PVT-SMVT patients usually go to the emergency department for unexplained abdominal pain 
or distension $(6,7)$. In order to achieve early diagnosis and treatment, emergency physicians should not ignore PVST, especially to judge the severity of embolism and whether it has caused intestinal necrosis. Discriminating between patients with PVST who developed intestinal necrosis and are in urgent need of operation from patients who have reversible intestinal congestion that can be managed with conservative measures can serve to decrease the morbidity and mortality rates and improve the outcome of patients with PVST.

In order to facilitate decision making in patients with PVST, we designed this study to determine the potential clinical and laboratory factors associated with intestinal necrosis and to construct a novel model based on these factors to predict the occurrence of intestinal necrosis in patients with PVST.

\section{Methods}

\section{Patients}

Records of patients with PVST who were admitted to the Department of Emergency Medicine, First Affiliated Hospital, Zhejiang University School of Medicine, from January 2010 to October 2018 were retrospectively reviewed.

Patients who presented to the emergency department with symptoms and signs of acute abdominal condition were admitted and investigated. Patients with chronic mesenteric ischemia without acute intestinal injury, patients with acute abdominal conditions or intestinal obstruction secondary to causes other than PVST, and patients with incomplete records missing some of the vital data required for the study were excluded.

PVST was diagnosed by a characteristic abdominal enhanced CT scan and exclusion of tumor embolism.

According to whether patients suffered from intestinal necrosis, all of the enrolled patients were divided into necrosis and non-necrosis groups.

Ethical approval of the study was obtained from the Ethics Committee of the First Affiliated Hospital, Zhejiang University School of Medicine, the current regulation of the Chinese Government and the Declaration of Helsinki. Every precaution has been taken to protect the privacy of research subjects and the confidentiality of their personal information. Informed consent was obtained from all patients.

\section{Treatment}

\section{Exploratory laparotomy}

The patients with deteriorating clinical condition or CT-confirmed intestinal necrosis received exploratory laparotomy. During exploratory laparotomy, the bowel viability was checked. If the bowels were found to be ischemic yet still viable, they were returned to the peritoneal cavity, and the abdomen was closed. In contrast, if nonviable bowel loops were found, resection with primary anastomosis was then undertaken.

\section{Thrombolytic therapy}

If the duration from onset of abdominal pain to hospitalization was $<14$ days, thrombolytic therapy was given.

\section{Conservative treatment}

Stable patients were treated conservatively. Conservative treatment comprised the administration of intravenous fluids guided by a fluid chart, correction of arterial blood gas (ABG) and electrolyte disturbances, intravenous antibiotics ( $2 \mathrm{~g}$ of third generation cephalosporines every $12 \mathrm{~h}$ and $500 \mathrm{mg}$ of ornidazole every $24 \mathrm{~h}$ ), and subcutaneous injection of low-molecular-weight heparin calcium (Fraxiparine $4100 \mathrm{U}$ ) every $12 \mathrm{~h}$.

\section{Data collection}

The following variables were recorded for the study population: age, sex, etiology, operation history, clinical manifestation, complete blood count (CBC), liver function, kidney function, $\mathrm{ABG}$ analysis result, coagulation function, venous embolization site, and therapy. All data from the laboratory and radiological examinations performed within 30 minutes after the patient arrived at emergency medicine were collected.

\section{Statistical analysis}

The statistical analysis was performed using the statistical software IBM SPSS software (Ver. 19.0; SPSS Inc., Chicago, IL, USA). Quantitative variables are expressed as the mean \pm standard deviation or median and range depending on the distribution. Categorical variables are presented as values and percentages. Student's $t$-test was used to compare quantitative variables. The Chi-square test 
was used to compare categorical variables. To identify risk factors for intestinal necrosis, a multivariate analysis was performed using the logistic regression model with forward elimination. Receiver operating characteristic (ROC) curves were constructed to determine the optimal cut-offs of continuous factors in predicting recurrence. All tests were two-sided, with a $\mathrm{P}$ value of $<0.05$ considered statistically significant.

\section{Results}

\section{Overview}

Sixty-nine adult patients were included in the study. All of these enrolled patients were admitted to our emergency department because of abdominal pain. A total of 14 (20.3\%) patients developed radiologic or operative confirmed transmural intestinal necrosis. Patients included 42 (60.9\%) males and 27 (39.1\%) females. The mean age of patients was $46 \pm 13.8$ (range, $17-79$ ) years. Nineteen (27.5\%) patients had liver cirrhosis, 12 (17.4\%) had a history of splenectomy, and $5(7.2 \%)$ had a history of gastrointestinal surgery. Regarding clinical signs on admission, 35 (50.7\%) patients had signs of abdominal distention, 16 (23.2\%) had signs of intestinal obstruction, $4(5.8 \%)$ had ascites, and 3 (4.3\%) had hematochezia.

\section{Clinical factors related to intestinal necrosis}

The univariate results shown in Table 1 corresponded to the incidence of transmural intestinal necrosis. Compared with the patients without intestinal necrosis, the intestinal necrosis group showed significantly higher leukocyte counts, neutrophil-to-lymphocyte ratio (NLR), serum aspartate aminotransferase (AST), creatinine (Cr) and blood urea nitrogen (BUN) levels $(17.83 \pm 8.05 \times 10 \mathrm{E} 9 / \mathrm{L}$ vs. $9.55 \pm 5.20 \times 10 \mathrm{E} 9 / \mathrm{L}$, $\mathrm{P}<0.01 ; 15.72 \pm 2.65 \times 10 \mathrm{E} 9 / \mathrm{L}$ vs. $6.89 \pm 0.71 \times 10 \mathrm{E} 9 / \mathrm{L}, \mathrm{P}<0.01$; $50.93 \pm 15.35$ vs. $27.57 \pm 2.65 \mathrm{U} / \mathrm{L}, \mathrm{P}<0.05 ; 80.85 \pm 11.42$ vs. $63.08 \pm 2.36 \mu \mathrm{mol} / \mathrm{L}, \mathrm{P}<0.05 ; 6.93 \pm 2.65$ vs. $4.72 \pm 1.93 \mathrm{mmol} / \mathrm{L}$, $\mathrm{P}<0.01$ ) (Figures 1,2). There were no differences in $\mathrm{ABG}$ analysis results or coagulation function between the necrosis group and the non-necrosis group (Figures 3,4).

\section{Prediction model for intestinal necrosis (PMIN)}

Leukocytes, NLR, AST, creatinine and BUN were significantly correlated with intestinal necrosis in univariate analysis and were then entered into multivariate logistic regression (Table 2). A PMIN was established: PMIN score $=-5.476+0.356 \times$ serum BUN $(\mathrm{mmol} / \mathrm{L})+0.165 \times$ leukocyte $\times 10^{-9}$. The area under the curve (AUC) of the entire model was 0.843 (95\% CI, 0.699-0.987), with a standard error of 0.073 within 30 minutes after the patients arrived at our emergency medicine department for predicting the incidence of intestinal necrosis (Figure 5). Using the ROC curve, the cut-off value of PMIN predicting the onset of intestinal necrosis was -0.92 with a sensitivity and specificity of $78.6 \%$ and $90.4 \%$. According to their PMIN scores, all patients were divided into a high-risk group (PMIN score $\geq-0.92, \mathrm{n}=16$ ) and a low-risk group (PMIN score $<-0.92$, $\mathrm{n}=53)$. The proportion of intestinal necrosis in the highrisk group was much higher than that in the low-risk group (68.8\% vs. $5.7 \%, \mathrm{P}<0.01)$.

\section{Discussion}

PVST can lead to venous pressure increase and intestinal wall congestion, which can cause a state of reversible intestinal wall congestion that, if not promptly corrected, may progress to irreversible tissue damage and necrosis (8). The outcome of PVST depends on the development or extent of irreversible intestinal necrosis, which requires immediate resection of affected intestines (9). In particular, the early diagnosis of intestinal necrosis is crucial for planning the surgery. To aid in predicting PVST patient intestinal necrosis as early as possible, a time-point of 30 minutes after the patient arrives in emergency medicine was selected as the appropriate time for model construction.

In order to determine the predictors of intestinal necrosis, we divided the patients according to intestinal survival into two groups: patients with viable intestine and patients with intestinal necrosis. Transmural intestinal necrosis was diagnosed intraoperatively and confirmed by histopathologic examination of the resected nonviable loops. This study demonstrated that high BUN and leukocyte levels were two independent risk factors for the occurrence of intestinal necrosis in patients with PVST.

BUN is regarded as one of the most efficient kidney function parameters (10). The main causes of an increase in BUN are a high protein diet, decreased glomerular filtration rate and blood volume, congestive heart failure, gastrointestinal hemorrhage, fever, and increased catabolism. Koutroumpakis et al. found that a rise in BUN at $24 \mathrm{~h}$ may be the optimal marker in predicting pancreatic necrosis in acute pancreatitis (11). 
Table 1 Characteristics of patients with necrosis and without necrosis

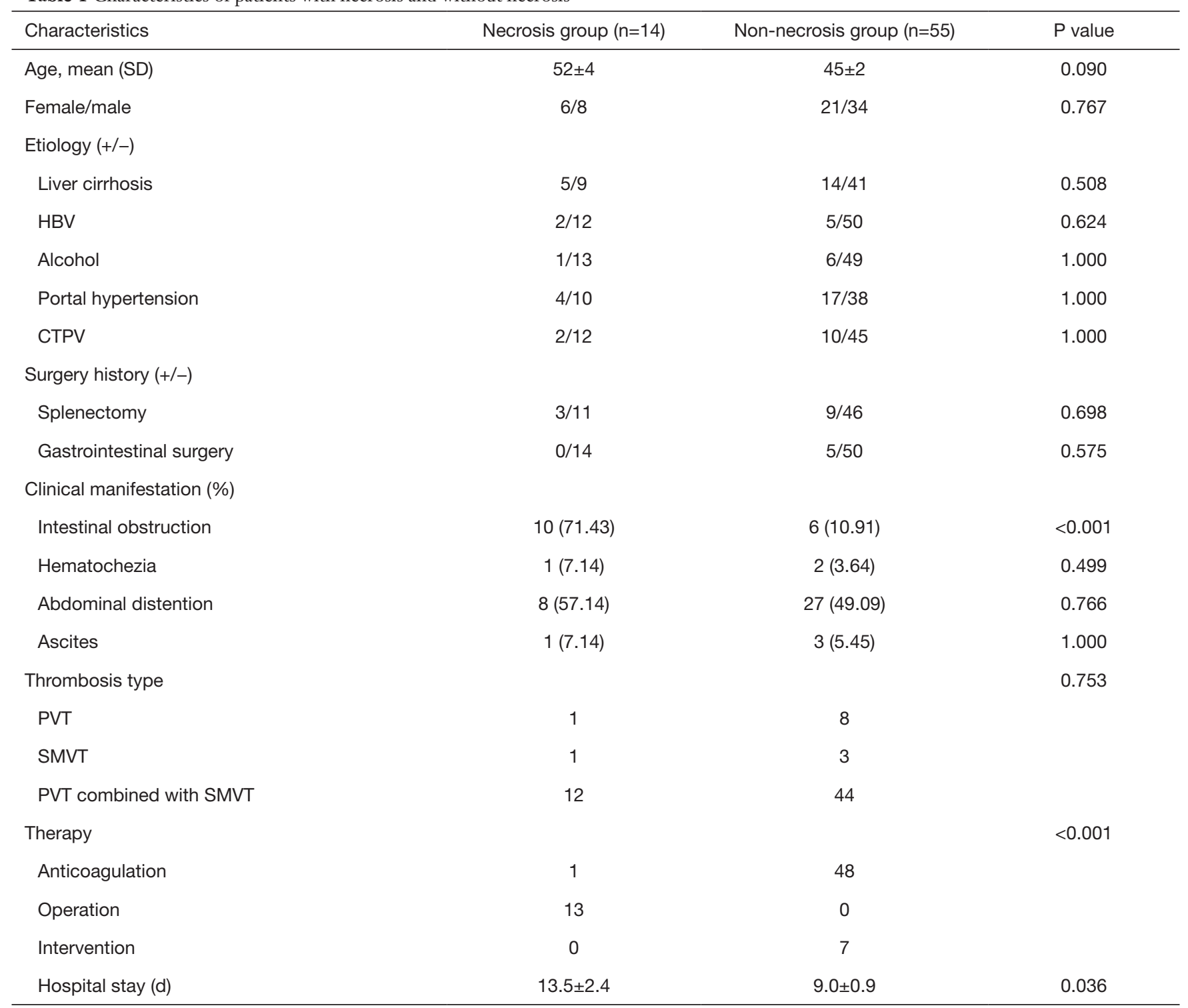

CTPV, cavernous transformation of portal vein; CRP, C-reactive protein; AST, aspartate aminotransferase; Hb, hemoglobin; APTT, Activated partial thromboplastin time; PT, prothrombin time; INR, international normalized ratio.

Some hemocytes, such as leukocyte counts, platelet counts and neutrophil ratios, are recognized and often applied as hematological inflammatory markers. In particular, the NLR and platelet-to-lymphocyte ratio (PLR) have been recently used as hematological inflammatory markers. The increase in neutrophil count during inflammation is recognized, which leads to relative lymphopenia in this process. Some previous studies have used the NLR as an important biomarker for predicting acute intestinal necrosis or acute mesenteric ischemia and the need for intestinal resection in patients who will undergo surgery for acute abdominal pain with the lack of radiological imaging methods (12-14). In the present study, leukocytes but not the NLR or PLR significantly predicted the onset of intestinal necrosis in patients with acute intestinal ischemia caused by PVST. Although the onset of intestinal ischemia is usually associated with leukocytosis (15-17), patients who developed intestinal necrosis in this study had a significantly higher leukocyte count than that of patients without intestinal necrosis. The 

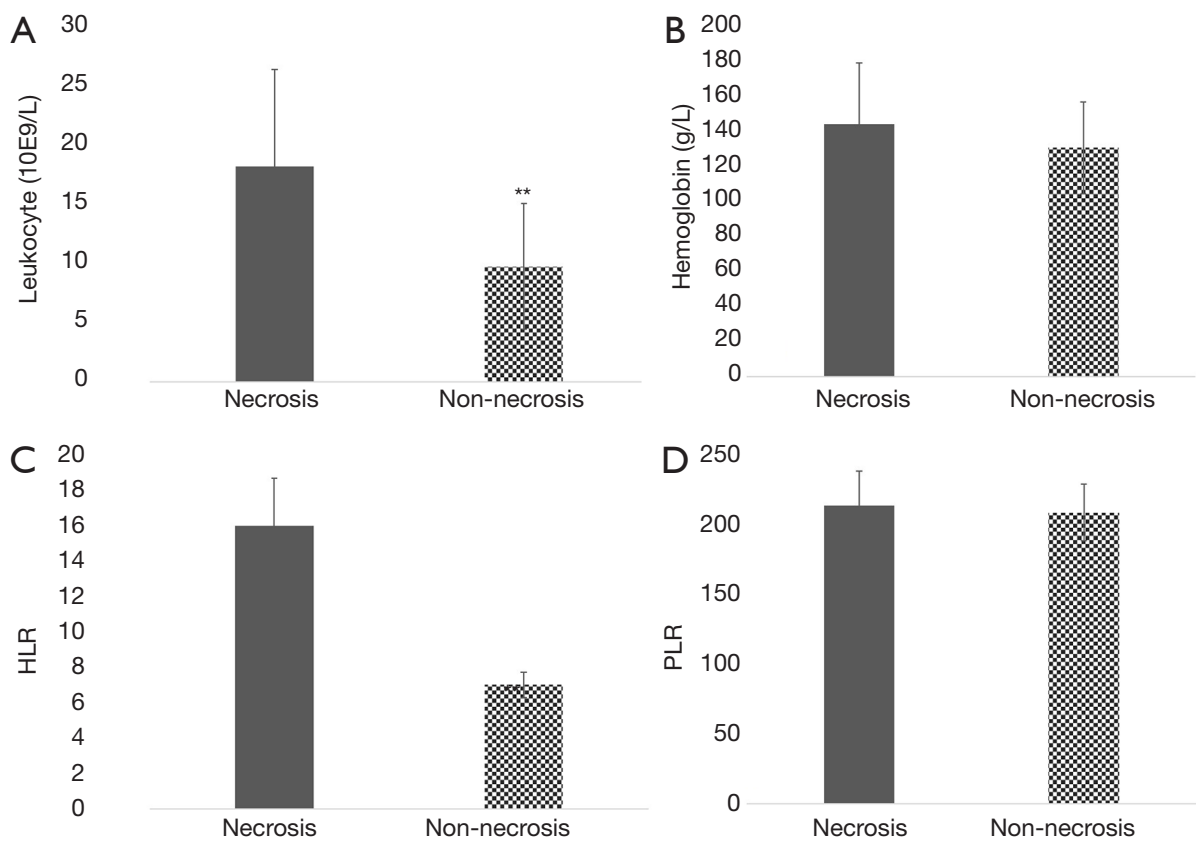

Figure 1 Comparison of the complete blood count (CBC) between the two groups. Compared with the patients without intestinal necrosis, the intestinal necrosis group showed significantly higher leukocyte counts (A) and neutrophil-to-lymphocyte ratios (NLRs) (C). $\left(17.83 \pm 8.05 \times 10 \mathrm{E} 9 / \mathrm{L}\right.$ vs. $9.55 \pm 5.20 \times 10 \mathrm{E} 9 / \mathrm{L}$ and $15.72 \pm 2.65 \times 10 \mathrm{E} 9 / \mathrm{L}$ vs. $6.89 \pm 0.71 \times 10 \mathrm{E} 9 / \mathrm{L},{ }^{* *}, \mathrm{P}<0.01$, respectively). Hemoglobin levels $(\mathrm{B})$ and the platelet-to-lymphocyte ratio (PLR) (D) did not differ significantly between the two groups $(\mathrm{P}>0.05)$.
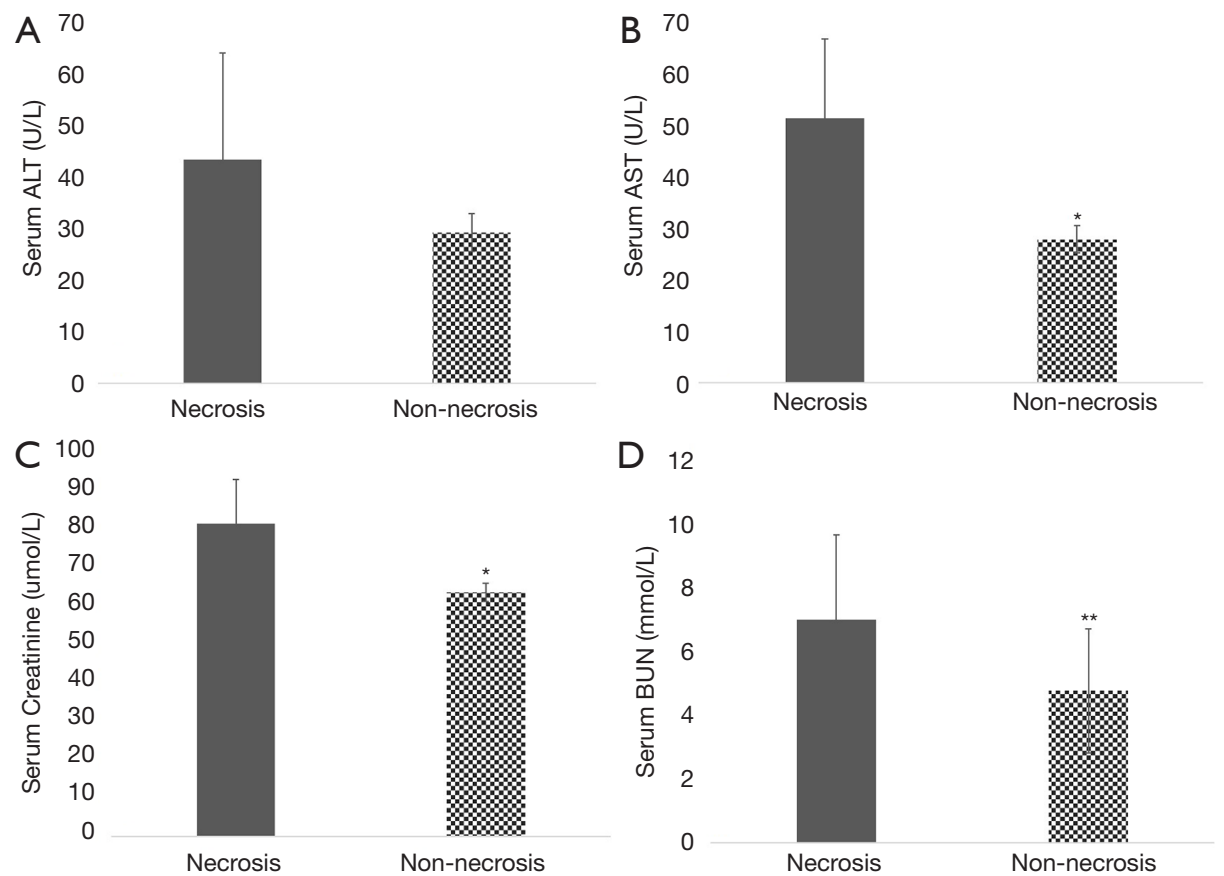

Figure 2 Comparison of liver and kidney function. Compared with the patients without intestinal necrosis, the intestinal necrosis group showed significantly higher serum aspartate aminotransferase (AST) (B), creatinine (Cr) (C) and blood urea nitrogen (BUN) (D) levels after being sent to emergency medicine. $\left(50.93 \pm 15.35\right.$ vs. $27.57 \pm 2.65 \mathrm{U} / \mathrm{L},{ }^{*}, \mathrm{P}<0.05 ; 80.85 \pm 11.42$ vs. $63.08 \pm 2.36 \mu \mathrm{mol} / \mathrm{L},{ }^{*}, \mathrm{P}<0.05 ; 6.93 \pm 2.65$ vs. $\left.4.72 \pm 1.93 \mathrm{mmol} / \mathrm{L},{ }^{* *}, \mathrm{P}<0.01\right)$. Alanine transaminase (ALT) (A) did not differ significantly between the two groups (P>0.05). 

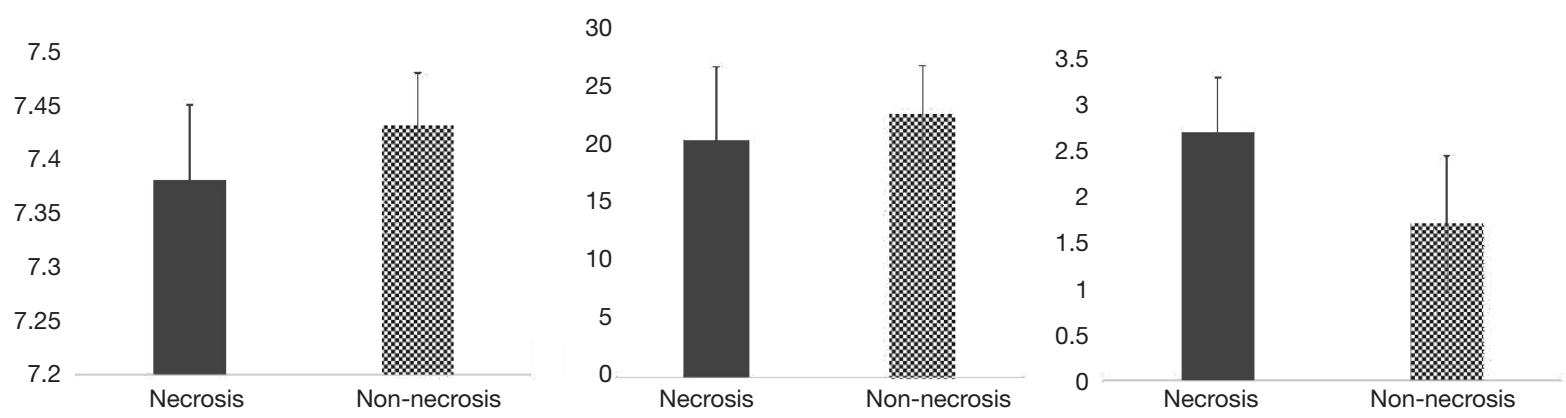

Figure 3 Comparison of arterial blood gas (ABG) analysis results between the two groups. There were no differences in the pH (A), bicarbonate concentration (B) and lactic acid concentration $(\mathrm{C})$ between the patients with and without intestinal necrosis $(7.38 \pm 0.07 v s$. $7.43 \pm 0.05,20.39 \pm 6.37$ vs. $22.65 \pm 4.21 \mathrm{mmol} / \mathrm{L}$ and $2.69 \pm 0.60$ vs. $1.70 \pm 0.74 \mathrm{mmol} / \mathrm{L}, \mathrm{P}>0.05$, respectively).
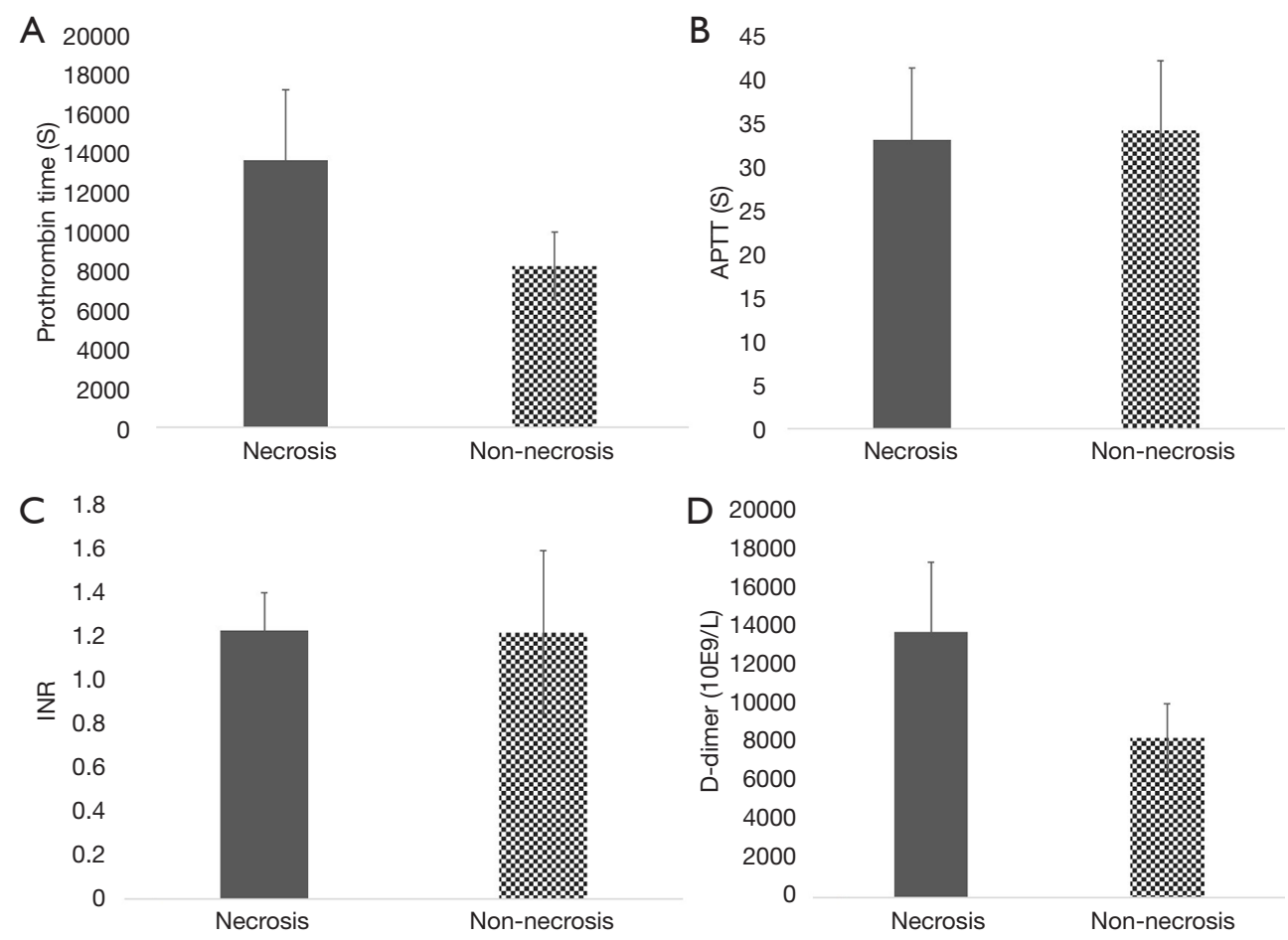

Figure 4 Comparison of coagulation function between the two groups. There were no differences in the prothrombin time (A), activated partial thromboplastin time (APTT) (B), international normalized ratio (INR) (C) and D-dimer (D) between the patients with and without intestinal necrosis $(14.11 \pm 2.09$ vs. $14.05 \pm 4.33 \mathrm{~s}, 33.12 \pm 8.26$ vs. $34.26 \pm 7.97 \mathrm{~s}, 1.22 \pm 0.17$ vs. $1.21 \pm 0.37$ and $13621.25 \pm 3586.99 \mu \mathrm{g} / \mathrm{L}$ vs. $8,202.21 \pm 1,736.91 \mu \mathrm{g} / \mathrm{L}, \mathrm{P}>0.05$, respectively).

Table 2 Multivariable analysis of factors associated with necrosis

\begin{tabular}{lcccccc}
\hline Variable & P value & B & SE & Wald Chi-square & OR & 95\% Cl for OR \\
\hline BUN & 0.048 & 0.356 & 0.175 & 3.909 & 1.413 & $1.003-1.992$ \\
Leukocyte count & 0.005 & 0.165 & 0.059 & 7.961 & 1.180 & $1.052-1.324$ \\
\hline
\end{tabular}

BUN, blood urea nitrogen; SE, standard error; OR, odds ratio. 


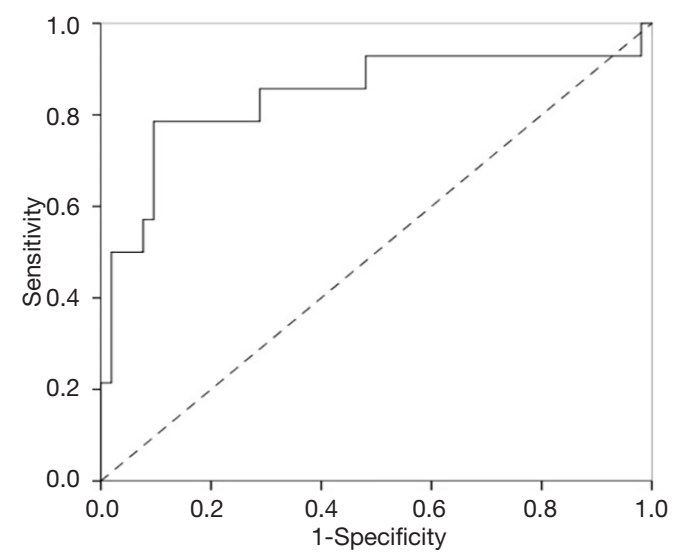

Figure 5 AUROC of the predicted model for intestinal necrosis (PMIN) for predicting the incidence of intestinal necrosis. The PMIN score was calculated within 30 minutes after the patients arrived in emergency medicine. The area under the curve of the entire model was 0.843 (95\% CI, 0.699-0.987), with a standard error of 0.073 .

higher leucocyte count in patients with intestinal necrosis could be interpreted that leukocytosis associated with reversible intestinal ischemia is considered one part of the systemic inflammatory response to ischemic injury, whereas intestinal necrosis is characterized by more bowel tissue injury and is accompanied by bacterial translocation, which may substantially increase the total leukocyte count (18).

The PMIN score based on serum levels of BUN and the leukocyte count has revealed a powerful prognostic ability, with an excellent AUC of 0.843 in retrospective studies and perfect predictive accuracy in prospective identification. Generally, many previous studies have focused on inflammatory biomarkers, such as the leukocyte count, neutrophils, and platelets, to predict intestinal resections. Some biomarkers were not taken into consideration. Our model is the first to focus on both inflammation and kidney function. Regardless of how perplexing the circumstances are, intestinal resections could be simply and accurately evaluated by only two variables immediately.

However, considering the limitation of sample size, potential bias may exist in this study. In order to gain a reliable conclusion, larger sample size or multicenter validation will be enrolled in the following years in our next research program.

In conclusion, the PMIN score is a simple and effective model based on BUN and the leukocyte count that can predict intestinal necrosis in patients with PVST.

\section{Acknowledgments}

Funding: This work was supported by the Changjiang Scholar Program of Chinese Ministry of Education (Grant Number T2014146) and the National Natural Science Funds for Distinguished Young Scholar of China (Grant Number 81625003).

\section{Footnote}

Conflicts of Interest: The authors have no conflicts of interest to declare.

Ethical Statement: The authors are accountable for all aspects of the work in ensuring that questions related to the accuracy or integrity of any part of the work are appropriately investigated and resolved. Ethical approval of the study was obtained from the Ethics Committee of the First Affiliated Hospital, Zhejiang University School of Medicine, the current regulation of the Chinese Government and the Declaration of Helsinki (No. 2013). Informed consent was obtained from all patients.

Open Access Statement: This is an Open Access article distributed in accordance with the Creative Commons Attribution-NonCommercial-NoDerivs 4.0 International License (CC BY-NC-ND 4.0), which permits the noncommercial replication and distribution of the article with the strict proviso that no changes or edits are made and the original work is properly cited (including links to both the formal publication through the relevant DOI and the license). See: https://creativecommons.org/licenses/by-nc-nd/4.0/.

\section{References}

1. Wolter K, Decker G, Kuetting D, et al. Interventional Treatment of Acute Portal Vein Thrombosis. Rofo 2018;190:740-46.

2. Quarrie R, Stawicki SP. Portal vein thrombosis: What surgeons need to know. Int J Crit Illn Inj Sci 2018;8:73-7.

3. Harding DJ, Perera MT, Chen F, et al. Portal vein thrombosis in cirrhosis: Controversies and latest developments. World J Gastroenterol. 2015;21:6769-84.

4. Cruz-Ramón V, Chinchilla-López P, Ramírez-Pérez $\mathrm{O}$, et al. Thrombosis of the Portal Venous System in Cirrhotic vs. Non-Cirrhotic Patients. Ann Hepatol 2018;17:476-81.

5. Mamode N, Pickford I, Leiberman P. Failure to improve 
outcome in acute mesenteric ischaemia: seven-year review. Eur J Surg 1999;165:203-8.

6. Garcia-Pagán JC, Hernández-Guerra M, Bosch J. Extrahepatic portal vein thrombosis. Semin Liver Dis 2008;28:282-92.

7. Liu FY, Wang MQ, Fan QS, et al. Interventional treatment for symptomatic acute-subacute portal and superior mesenteric vein thrombosis. World J Gastroenterol 2009;15:5028-34.

8. Wang CY, Wei LQ, Niu HZ, et al. Agitation thrombolysis combined with catheter-directed thrombolysis for the treatment of non-cirrhotic acute portal vein thrombosis. World J Gastroenterol 2018;24:4482-8.

9. Brandt LJ, Boley SJ. AGA technical review on intestinal ischemia. American Gastrointestinal Association. Gastroenterology 2000;118:954-68.

10. Wesseling C, Aragón A, González M, et al. Kidney function in sugarcane cutters in Nicaragua--A longitudinal study of workers at risk of Mesoamerican nephropathy. Environ Res 2016;147:125-32.

11. Koutroumpakis E, Wu BU, Bakker OJ, et al. Admission Hematocrit and Rise in Blood Urea Nitrogen at $24 \mathrm{~h}$ Outperform other Laboratory Markers in Predicting Persistent Organ Failure and Pancreatic Necrosis in Acute Pancreatitis: A Post Hoc Analysis of Three Large Prospective Databases. Am J Gastroenterol

Cite this article as: Wei Q, He Z, Wang K, Li C, Al Ameri A, Zheng S, Xu X. Prediction model based on blood urea nitrogen and the leukocyte count for intestinal necrosis in patients with portal vein system thrombosis: a retrospective study. Ann Transl Med 2020;8(6):326. doi: 10.21037/atm.2020.02.108
2015;110:1707-16.

12. Aktimur R, Cetinkunar S, Yildirim K, et al. Neutrophilto-lymphocyte ratio as a diagnostic biomarker for the diagnosis of acute mesenteric ischemia. Eur J Trauma Emerg Surg 2016;42:363-8.

13. Hwang SY, Shin TG, Jo IJ, et al. Neutrophil-tolymphocyte ratio as a prognostic marker in critically-ill septic patients. Am J Emerg Med 2017;35:234-9.

14. Köksal H, Ateş D, Nazik EE, et al. Predictive value of preoperative neutrophil-to-lymphocyte ratio while detecting bowel resection in hernia with intestinal incarceration. Ulus Travma Acil Cerrahi Derg 2018;24:207-10.

15. Sise MJ. Mesenteric ischemia: the whole spectrum. Scand J Surg 2010;99:106-10.

16. Balaz P, Rokosny S, Bafrnec J. Mesenteric ischemia, new trends, diagnostic methods and algorithms to significantly reduce mortality and morbidity. Bratisl Lek Listy 2013;114:158-65.

17. Akyıldız HY, Sözüer E, Uzer H, et al. The length of necrosis and renal insufficiency predict the outcome of acute mesenteric ischemia. Asian J Surg 2015;38:28-32.

18. Kaplan LJ. Systemic inflammatory response syndrome. Medscape. 2017; Accessed 3 Nov 2017. Available online: http://emedicine.medscape.com/article/168943-overview 